\section{A V K}

\section{Bewerbungen zum Innovationspreis 2013}

Die AVK - Industrievereinigung Verstärkte Kunststoffe e.V. vergibt in diesem Jahr wieder vier Preise für Innovationen im Bereich Faserverstärkte Kunststoffe und bittet um Bewerbungen zum AVK-Innovationspreis 2013.

Ziel der Auszeichnung ist die Förderung neuer Produkte oder Bauteile beziehungsweise Anwendungen aus faserverstärkten Kunststoffen sowie die Förderung neuer Verfahren und Prozesse zur Herstellung dieser Produkte. Ein Preis geht an Universitäten, Hochschulen und Institute für herausragende wissenschaftliche Arbeiten in Forschung und Wissenschaft. Ein weiterer Preis wird an einen besonders attraktiven FVKArbeitgeber verliehen. Hier sind Personalmanagement-Leistungen, Unternehmenskultur sowie Arbeitssicherheit und Arbeitsschutz Bewertungskriterien. Ein wichtiges
Bewertungskriterium der Einreichungen ist auch das Thema "Nachhaltigkeit".

Der AVK-Innovationspreis soll Innovationen sowie die dahinterstehenden Personen und Institutionen auszeichnen und so die Leistungsfähigkeit der gesamten Branche an die Öffentlichkeit tragen. Einsendeschluss für die Bewerbungsunterlagen ist der 25. März 2013. Die Preisverleihung erfolgt anlässlich der Internationalen AVK-Tagung vom 16.17. September 2013 im ICS Internationalen Congresscenter Stuttgart, in Anbindung an die Fachmesse Composites Europe vom 17. bis 19. September 2013.

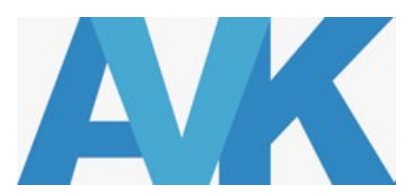

\title{
Zulassung des Germanischen Lloyd für
}

\section{SE2020-Verstärkungen}

3B, "the fibreglass company" im belgischen Battice, hat für seine neu entwickelten Single-End-Rovings vom Typ SE2020 die Zulassung des Germanischen Lloyd (GL) erhalten. Die Rovings werden vorwiegend in der Gelegefertigung für Rotorblätter von Windkraftanlagen eingesetzt.

"Gelege aus SE2020-Rovings eignen sich für Harzinfusions- und Prepregverfahren. Dabei profitieren sie von der innovativen und proprietären Schlichtetechnologie von 3B für spezifische Harzsystems, wie in diesem Fall Epoxidharz", sagt Luc Peters, Technischer Leiter bei 3B Wind Energy. Die resultierenden Laminate böten ein höheres Maß an Ermüdungsbeständigkeit so- wie überlegene Zwischenfaser- und interlaminare Scherfestigkeiten. Das mache sie zu einer Schlüssellösung für Konstrukteure, die mit der Entwicklung der nächsten Generation von Rotorblättern für Windkraftanlagen befasst sind.

Die SE2020-Rovings werden aus borfreiem E-CR-Glas gefertigt, das eine signifikant höhere Korrosionsbeständigkeit gegen eine Vielzahl aggressiver Umgebungseinflüsse biete. Dies und verbesserte Verbundwerkstoffeigenschaften kämen den Leistungsanforderungen an Rotorblätter vor allem in Offshore-Anlagen, wo extremere Betriebsbedingungen vorherrschen, optimal entgegen.
FASERVERBUND

NEUER AVK-ARBEITSKREIS

"REPARATUR VON

"COMPOSITES" GEGRÜNDET

Faserverstärkte Kunststoffe haben in den vergangenen Jahren Einzug in viele Anwendungsbereiche gefunden. Besonders im Fokus stehen dabei fast immer langlebige, industrielle Produkte und Einsatzmöglichkeiten. Aber auch darüber hinaus werden Composites oder entsprechenden Multimatrial-Systemen große Potenziale zugeschrieben.

Immer intensiver werden diese Konstruktionswerkstoffe in jüngster Zeit wegen des Einsatzes von CFK in der automobilen Großserie diskutiert. Speziell vor dem Hintergrund eines möglichen Serieneinsatzes, den Spezifika bisheriger Anwendungen, zum Beispiel in der Windenergie aber auch aufgrund seiner besonderen Eigenschaften, erhält die Entwicklung und Standardisierung entsprechender Reparaturmöglichkeiten in letzter Zeit einen zentralen Stellenwert.

Um den Erfahrungsaustausch der unterschiedlichen Marktakteure zu vorhandenen Reparaturmöglichkeiten und zukünftigen Methodiken zu gewährleisten, hat die AVK - Industrievereinigung Verstärkte Kunststoffe e.V. am 4. Dezember 2012 den neuen Arbeitskreis "Reparatur von Composites" gegründet. Unter der Leitung von Professor Dr. Jens Ridzewski (Bild), IMA Materialforschung und Anwendungstechnik GmbH in Dresden und AVK-Vorstandsmitglied, soll durch den gemeinsamen Austausch von zielgerichteten Lösungsansätzen der Einsatz von Composites in bestehenden und neuen Anwendungsbereichen gefördert werden.

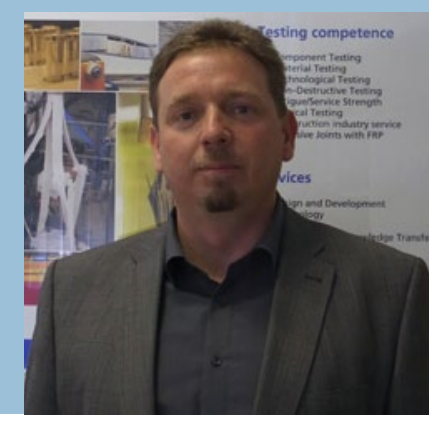

